\title{
ASPECTOS NARRATIVOS DE ALPHONSINE, OU LA TENDRESSE MATERNELLE DE MME DE GENLIS
}

\author{
Ángeles García Calderón \\ Universidad de Córdoba
}

\section{Resumen}

Se trata de un trabajo que versa sobre uno de los apólogos o narraciones parenéticas de Mme de Genlis, Alphonsine, ou la Tendresse maternelle, en el que la autora, siguiendo el modelo utilizado por Rousseau en Émile, aconseja a su hija para que aproveche las facultadas dadas por Dios al ser humano, exhortándola a que las utilice para ser feliz. Tras una breve descripción de la figura de Mme de Genlis, resaltando su importancia como novelista de relatos históricos y ensayos educativos, así como su influencia en Inglaterra y España, el trabajo se centra en un análisis pormenorizado de las fuentes, personajes, dinámica narrativa y espacio de la novela.

Palabras clave: Mme de Genlis, relatos apologéticos, estructura narrativa.

\section{NARRATIVE ASPECTS OF ALPHONSINE, OU LA TENDRESSE MATERNELLE BY MME DE GENLIS}

\begin{abstract}
This paper discusses Mme de Genlis's apologetic stories or narrations of Mme de Genlis, Alphonsine, or Maternal Affection, in which the author, following the model derived from Rousseau in Émile, advises her daughter to take advantage of the faculties given by God to human beings, exhorting her to apply them in order to be happy. After a brief description about the figure of Mme de Genlis, highlighting her importance as a novelist of historical stories and educational essays, as well as her influence in England and Spain, this work focuses on a detailed analysis of the sources, characters, narrative dynamics and space.

Keywords: Mme de Genlis, apologetic stories, narrative structure.
\end{abstract}

Fecha de recepción: 11 de septiembre de 2018

Fecha de aceptación: 13 de mayo de 2019 


\section{INTRODUCCIÓN}

Mme de Genlis es uno de los ejemplos más notables de esas mujeres escritoras que harán de su pluma una profesión; creadora en Francia junto con Mme Cottin de la novela histórica, antes de que con Dumas alcance cotas inimaginables, sus libros se editarán amplia y profusamente, destacando como una de las mujeres más prolíficas de su siglo cuya influencia en Europa, y sobre todo en Inglaterra, será enorme tanto entre los lectores como entre los propios escritores ingleses. Así por ejemplo, una autora tan traducida al francés como Jane Austen estimaba enormemente las Veillées $d u$ Château de Mme de Genlis ${ }^{1}$.

En España su obra Adèle et Théodore ou Lettres sur l'éducation contenant tous les principes relatifs à l'éducation des Princes, des jeunes personnes et des hommes (Paris, Lambert \& F.J. Baudouin, 1782) sería traducida sólo tres años más tarde, en $1785^{2}$, contándose desde esa fecha y durante todo el xix con múltiples traducciones de varias de sus obras, entre las que algunas son reiteradamente versionadas: Veladas de la quinta, Adelayda o el triunfo del amor, El zafir portentoso, La señorita de Clermont, Plácido y Blanca o Las Batuecas, Zuma o el descubrimiento de la quina, El sitio de la Rochela, Valeria y Beaumanoir o la caprichosa penitencia, La bella Paulina, o Amar sin saber a quién, Alfonso o el hijo natural, Las cañas del Tíber o Los desgraciados amores de Rozeval y Urania, Inés de Castro, Las madres rivales o La calumnia, El apóstata y la devota o sea el poder irresistible de los buenos principios, Los votos temerarios o El entusiasmo, El castillo de Kolmeras, etc. Junto con el hispanista Florian y Mme Cottin nuestra autora sería, a finales del xviıI y durante buena parte del xix, una de las escritoras más leídas y traducidas en España, país donde sus obras gozarían de una prodigiosa difusión ${ }^{3}$.

Pero, a pesar de todos los títulos reseñados, en España la obra de la que trata este trabajo parece haber sido prácticamente ignorada, ya que no hay vestigios de que Alphonsine no sólo no fuera traducida a nuestra lengua ${ }^{4}$, sino de que ni siquiera mereciera muchos comentarios al respecto, de ahí

1 En Inglaterra la traducción de Alphonsine tendría lugar el mismo año de su publicación en Francia (Genlis, 1806b). Asimismo, la novelista irlandesa especializada en cultura francesa Julia Kavanagh (1824-1877), en un volumen dedicado a las escritoras francesas, elogiaría enormemente este libro.

2 Adela y Teodoro o Cartas sobre la educación, traducida por don Bernardo María de Calzada.

3 Fernández Montesinos (1982: 198-200) dedica tres páginas y veintiséis entradas a las obras traducidas de Mme de Genlis entre 1785 y 1850.

4 Merecieron más atención los ensayos sobre educación infantil o las novelas puramente históricas, no entrando de lleno en este epígrafe Alphonsine, quizá por su mezcla de narración histórica y sentimental, o posiblemente por la sencilla dicotomía que encierra el relato como plasmación de un maniqueísmo en exceso simplista. 
la originalidad y posible relevancia de nuestro análisis, al dar a conocer las características de una obra que no desmerece en absoluto del resto de la producción de nuestra escritora ${ }^{5}$.

Alphonsine, ou la Tendresse maternelle, publicada en 1806 en dos volúmenes ${ }^{6}$, se incluye dentro de los apólogos o narraciones parenéticas ${ }^{7}$. Los rasgos lingüísticos y discursivos del texto se esbozan por medio de la función apelativa, constante en este tipo de obras, como si la autora quisiera influir en el destinatario orientando su opinión. En este aspecto, el propio título ya advierte de que sus observaciones no son meras divagaciones, sino que nacen con la intención de inculcar a sus hijos la necesidad de mantener un comportamiento digno y respetable, utilizando para ello continuamente el vocativo «ma fille». No en vano la obra contiene una brevísima «Épître dédicatoire» a Mme de Valence, segunda de sus hijas ${ }^{8}$.

Tras esta dedicatoria sigue un «Préface» en el que la autora analiza su obra, contrastándola con las opiniones de Rousseau sobre la educación, y en el que esboza la dificultad de hacer verosímiles los acontecimientos en la novela. Posteriormente, hay una breve disección en la que Genlis expresa su opinión sobre la prohibición de la lectura de novelas a los alumnos por parte de los maestros.

El prólogo justifica a la perfección el por qué y para quién está escrita la novela, como podremos apreciar por las siguientes premisas:

a) El hecho de ser una novela histórica, a la vez que religiosa, asegura el triunfo según los gustos de los lectores de esa época.

b) Para describir lo mejor posible aquello de lo que es capaz una madre, es decir, para explicar hasta qué punto puede desarrollar su capacidad de ternura maternal ha tenido que poner a las dos protagonistas en una situación extrema, lo que obliga a la madre a demostrar su gran capacidad de cariño.

c) El privar de la visión a Alphonsine era del todo lógico, ya que para la niña no representaba una carencia: para ver «realmente» hay que saber comparar, es preciso saber admirar.

5 Un breve pero interesante resumen sobre la autora y su obra puede encontrarse en Landy (1998), que aconseja como estudio de conjunto para la autora Laborde (1966). Aunque no se ocupan en exceso de la novela aquí estudiada, véanse el estudio ya clásico de Broglie (2001) y los más recientes de Barthélemy (2006) y Bessire y Reid (2008), que incide en la vertiente educativa de Genlis.

${ }^{6}$ En este trabajo citamos esta edición (Genlis, 1806a), indicando únicamente volumen y página. Existe otra edición de tres tomos, citada por Díaz Canseco (1844: II: 153). En esta edición se repite el capítulo xxir: último del primer tomo y primero del segundo.

7 Lo que no excluye que puedan incluirse dentro del género ensayístico, como tratados de educación que son.

8 Louise Josephine Philippine Félicité Séraphine de Timbrune de Valence (1787-1828). 
d) El dotar a la niña de todo el candor, pureza e inocencia posibles exigía que se concentrara absolutamente en la madre, manteniéndola alejada de la pasión de un amor.

e) La intención final de la novela, así como el motivo de su composición, es moral; por ello, el mostrar a las almas en la situación más deplorable e inhumana propiciaba que estas debieran utilizar todos los recursos a su alcance para poder soportar la situación.

La obra está formada por cincuenta capítulos: veintidós en el primer tomo y veintiocho en el segundo. La distribución pone de relieve que no todos los capítulos tienen una extensión similar, ya que podemos hallar algunos de mucha más longitud que otros; así, tras presentar a los personajes y acontecimientos principales, el capítulo ix, dedicado a Dazeli y en el que el joven expone al rey cómo ocurrieron los hechos de su falsa huida con Diana, comprende desde la página 50 a la 131; los dos últimos capítulos del primer tomo, xxi y XxiI (págs. 195-286 y 287-372) y el primero del segundo tomo (capítulo xxII, págs. 1-163) están dedicados a la narración de la historia de Diana en prisión, el nacimiento y la educación de su hija hasta el momento en que son liberadas.

Un análisis pormenorizado de las fuentes, los personajes, la dinámica narrativa y el espacio de la novela pondrán de relieve la importancia de la educación para la escritora.

\section{Fuentes e influencias (Mme de Genlis y su cultura novelística)}

Imbuida de la cultura de siglos pasados, la obra tiene reminiscencias de narradores franceses de otras épocas, ya que Genlis es deudora de novelistas y escritores anteriores, entre los que podemos citar:

a) Los narradores del XviI francés: diríase que los amantes deben pasar por las pruebas que prescribía Mlle de Scudéry en su "Carte du Tendre», incluida en Clélie, Histoire romane, para llegar a alcanzar el amor de su dama.

b) La escena en la que don Sanche propicia que Diana, escondiéndose en un «cabinet», pueda percibir en toda su crudeza el carácter malévolo de su esposo, el conde, es similar a la escena de la confesión de la Princesa de Clèves a su marido, sin que ella sepa que su enamorado Nemours está oyéndola escondido.

c) Se inspira claramente en Laclos en lo que concierne al vicio y la depravación, como se deduce por la conversación entre Elvire y D. Fernand ${ }^{9}$ :

9 Dada la fecha de publicación de la obra, 1806, he mantenido la grafía original, modernizando únicamente los finales de imperfecto en oi, ô por las actuales terminaciones. 
D. FERnAND

Convenez qu'il est un peu bête?

Elvire

Mais pas trop; c'est un enfant...

\section{Fernand}

Formé par vous il devrait être aimable.

Elvire

Pour le conserver, j'ai voulu lui donner des principes, et j'en ai fait un sot...

\section{Fernand, riant}

Amusez-vous à le pervertir; alors vous reprendrez du goût pour lui.

\section{Elvire, riant}

Non. J'aimerais mieux un autre élève; car une éducation mal commencée ne finit jamais bien (I: 123-124).

d) Hay una clara alusión al final de Candide de Voltaire, al citar en cursiva: le plaisir de cultiver son jardin (I: 141).

e) La lectura del manuscrito que relata la historia de doña Diana de Mendoce tiene claras reminiscencias de las novelas góticas inglesas por la minuciosa descripción de puertas, escaleras, pasillos, cuevas, galerías y subterráneos del castillo del que intenta evadirse Diana, al poco tiempo de ser encerrada (I: 287-295).

A la vez que la autora se inspira en escritores anteriores, ella a su vez inspiraría a escritores posteriores:

a) A Dumas padre, por la similitud del complot urdido por Don Sanche para hacer salir de Madrid a Diana y su paje, similar al urdido contra Edmundo Dantés en Le Comte de Monte-Cristo.

b) A Stendhal en La Chartreuse de Parme, en un pasaje similar a la escena de Fabricio en la Torre Farnesio, enamorado de Clélia Conti: la prisión = lugar de felicidad, que se ha convertido en un verdadero calabozo para la heroína de Genlis.

Pénétré de mépris, et saisi d'horreur, je sortis enfin du cabinet; je m’échappai sans bruit, et je regagnai ma tour, qui, tout-à-fait désenchantée, ne me parut plus alors qu'une insupportable prison... (I: 126-127).

c) A Walter Scott: la muerte del conde de Moncalde, delirando e identificando a su gobernanta Léonore con un monstruoso fantasma, es similar a la de Front-de-Bœuf delirando y viendo enfrente a su antigua amante Ulrica, en el capítulo xxx de Ivanhoe. 


\section{Retratos (CARActeres) $)^{10}$}

La descripción de cada uno de los personajes principales revela al lector cuáles son sus vicios o virtudes; así, basándonos en la simple distinción maniquea que utiliza la autora, veamos los caracteres de unos y otros:

a) El Conde de Moncalde:

Lorgueil était le seul mobile de ses actions, et la véritable source de ses vices et de ses qualités sociales. Son ambition n’avait pour motif que le désir de paraître et de briller. Sa cupidité n'était que l'amour du faste, et son goût pour les femmes que de la fatuité; frivole et léger, autant que pervers, il n'était susceptible d'aucune grande passion (I: 13).

La propia confesión del conde y la de Léonore, así como las narraciones de Dazeli y del cura, van plasmando cada vez más el carácter del conde.

El conde piensa de su joven esposa que es insípida y boba, torpe y sin ingenio; además, tiene el «defecto» de estar enamorada de él.

b) Don Sanche de Mélez: raptor de Diana (de acuerdo con el conde de Moncalde, a su vez tiene una hija, Aurora, la cual inspiró una pasión asesina a un joven que el padre de Aurora había recogido: Mulzino. Don Sanche se inspira en la escapatoria de Aurora y Mulzino, terminada trágicamente, para raptar a Diana. Su retrato y carácter son descritos en el capítulo tercero:

Ce dernier avait infiniment plus d'esprit que le comte. Ses passions étaient violentes, et nuls principes n'en tempéraient en lui l'impétuosité. S'il n'eût pas été corrompu dès sa première jeunesse, il aurait pu avoir d'éminentes

10 Por lo que respecta a los nombres de la novela, Hervey y Higgins (1992: 29) señalan que, en principio, pueden establecerse dos alternativas: o bien el nombre de la lengua origen se mantiene sin alterar en la lengua meta, o bien se adapta conforme a las convenciones fónicas y gráficas de la lengua meta. La primera forma se correspondería con una traducción «exotizante» o «extranjerizante», aunque en algunos casos es impracticable (en los nombres rusos, por ejemplo). En cuando a la segunda alternativa, la transliteración, es menos extrema, aunque cómo se lleve a cabo la transliteración depende del traductor (en los casos en que no exista un precedente establecido o acuñado). Más tarde, plantean una tercera opción: el trasplante cultural (cultural transplantation), que constituye el grado extremo de transposición cultural, y tiene lugar cuando un nombre propio de la lengua origen se sustituye por un nombre propio en la lengua meta sin que sean equivalentes literales, aunque compartan connotaciones culturales. En el caso que nos ocupa, el relato de Mme de Genlis, procede una aclaración: la autora ha utilizado nombres franceses aunque la acción se desarrolle enteramente en España, de ahí que a veces nos parezca un anacronismo utilizar Alvar en lugar de Álvaro, Alphonsine en lugar de Alfonsa (digamos, de paso, que es un error mayúsculo la denominación de María Antonieta para referirse a la reina francesa, cuando debiera ser María Antonia; por la misma razón, Alphonsine —-femenino de Alphonse- debería ser Alfonsa); el anacronismo es aún mayor al escribir Inès por Inés, cuando en francés el nombre es Agnès. Así pues, para evitar cualquier tipo de confusión, respetaremos la grafía original de los nombres. 
qualités, avec un grand caractère; mais, dès les premiers pas de sa carrière, engagé dans les routes du vice, il s'y était fixé sans retour, en épuisant tous les excès (I: 15).

c) Léonore, la gobernanta del conde de Moncalde:

Léonore, prude, bigote, superstitieuse, intolérante et vindicative, faisait consister toute la religion d'une femme à n'avoir point d'amour, et à suivre scrupuleusement une infinité de petites pratiques, que l'église autorise sans les prescrire, et qui n'ont de prix aux yeux de la religion que lorsqu'elles sont accompagnées des vertus évangéliques (I: 19).

En palabras de Dazeli es una gobernanta inflexible; en el capítulo ix se ven sus maquinaciones y oscuros secretos, así como sus artimañas de espía y delatora. Si Léonore llega a salvar su vida, después de tantas crueldades, es gracias a sus ideas religiosas que hacen que Diana la perdone y disculpe a veces:

Léonore, loin d'être impie, eut toujours des idées religieuses; connaissant mal la religion, elle ne s'est jamais élevée jusqu'à l'amour de Dieu, mais elle en a la crainte. Je dois à cette crainte salutaire et mon existence et celle de mon enfant (II: 102).

d) Los criados del conde de Moncalde son, según las palabras de Léonore, sinvergüenzas, borrachos y ladrones:

Je suis maîtresse de congédier les domestiques à mon gré; les servantes que j’ai trouvées en arrivant dans ce château, étaient des coquines, les valets des ivrognes et des voleurs, et j'ai fait maison nette (I: 27).

e) Elvire, la viuda del gobernador del castillo de Cádiz donde ha estado encerrado Dazeli: de espiritual, «aimable et piquante» (I: 113), cuando muestra su verdadera faz es la viva representación del vicio y la perversidad:

Enfin cette personne, dont l'entretien venait de me dévoiler la profonde dépravation, se dépouillant encore de tous ses agréments extérieurs, ne m'offrait plus que l'affreuse image et la dégoûtante réunion du vice et de la vieillesse... (I: 126).

No obstante, en el momento de morir, apela al socorro de la religión:

Elvire, remplie de remord et de terreurs, n'attendant plus rien des secours humains implorait ceux de la religion, et refusa de me recevoir (I: 129).

Por la parte de los personajes virtuosos:

a) El duque de Mendoce, abuelo de Diana y tío de Moncalde: solo aparece en la novela por referencias, pero es siempre descrito como un hombre 
de honor, justo, recto y de buenos sentimientos. Personaje al que todos los demás admiran, es dibujado en varios pasajes como un hombre virtuoso; en su palacio Diana pasaría felizmente su infancia.

b) La duquesa de Olmas, hermana de Don Pèdre de Almédor y segunda esposa de Don Pèdre de Moncalde: «Elle est très vertueuse et très-bonne» (I: 26). Aparentemente, por sus cualidades, es un trasunto de Mme de Genlis, aunque en el capítulo xxxvin se muestre en total desacuerdo con el modo de educar a Alphonsine:

Alors la comtesse instruisit don Alvar de ce que lui avait dit Diana. Don Alvar eut beaucoup de peine à dissimuler le chagrin extrême que lui causait cette défense de Diana. La comtesse aussitôt changea d'entretien. Elle parlait rarement de Diana, dont elle n'approuvait ni la manière de vivre ni les systèmes. Les esprits sages, mais médiocres, ont un éloignement naturel pour tous ceux dont les idées diffèrent des idées reçues; ils ont raison en général; mais lorsqu'il faudrait admettre des exceptions, ils ne sont pas en état de les faire (II: 296-297).

c) Dazeli, que huye siendo paje con Diana y luego hará fortuna cerca del rey, casándose finalmente con Inès:

Dazeli joignait à une figure charmante un naturel plein de grâces, une franchise qui lui gagnait tous les cœurs, et la gaieté la plus aimable. Il avait si peu de prétentions, tant de vivacité dans l'esprit et de simplicité dans les manières, que le manque total d'usage du monde n'était en lui qu'un charme de plus; il donnait à cette espèce d'ignorance une naïveté intéressante, et la plus piquante originalité (I: 50).

Cuando Don Sanche lo hace desaparecer con el nombre de Mulzino de Sevilla, o Edmond, no reaparece hasta ser amigo del primer ministro.

d) Don Pèdre de Almédor, hermano de la duquesa de Olmas y padre de Alphonsine, que finalmente se casará con Diana, es descrito por Dazeli, al contar éste su historia al rey, y luego por la propia Diana:

Je n'avais que quatorze ans lorsque don Pèdre d'Almédor me prit chez lui en qualité de page. Je me trouvai heureux de servir un jeune maître rempli d'agrémens, d'esprit et de bonté, et qui n'avait que huit ans de plus que moi. Don Pèdre s'occupa de mon éducation, il me donna d'excellents maîtres, il me combla de bienfaits, et il s'acquit autant de droits à ma reconnaissance qu'il en avait à mon amitié par ses qualités attachantes (I: 51).

e) Diana, casada con el conde de Moncalde, encerrada durante trece años en un subterráneo, madre de Alphonsine y casada finalmente con el padre de ésta, Don Pèdre de Almédor, es descrita con simpatía por la duquesa de Olmas, que finaliza con una reflexión muy acertada sobre el sentimiento 
de la melancolía, muy cercana a la atinada definición que encontramos en buena parte de la literatura francesa del siglo xix de la melancolía, como «maladie de langueur»:

Le lendemain, Diana, levée, ainsi qu'Alphonsine, reçut la comtesse. Cette dernière examinait, avec autant de surprise que d'attendrissement, Diana, qu'elle avait vue jadis si fraîche, si brillante, si animée, dont les manières étaient si affectueuses; maintenant, belle encore, mais décolorée, silencieuse, rêveuse, immobile, et d'une mélancolie douce et calme, qui ressemblait moins à la tristesse qu'au recueillement (I: 191).

Diana representa, sobre todo a ojos de su cruel enamorado Don Sanche, la perfección y la virtud; en el momento de enterarse de que es madre, éste habla de ella del siguiente modo:

O prodige de la tendresse maternelle! s'écria-t-il!... amour d'une mère! oui, tu surpasses tout autre sentiment; tu ne produis que des vertus sublimes. Infortunée Diana! que n'avez-vous pas souffert!... Vous êtes devenue mère dans cette caverne?... (II: 80-81).

¿Qué mayor prueba de amor y ternura podría dar a su hija Diana tras la liberación de ambas que no contemplar las creaciones de Dios, el cielo y la naturaleza hasta que no lo haga la propia Alphonsine en el día de su bautismo, hecho que simboliza a la perfección un nuevo nacimiento?

f) Alphonsine: educada por su madre en el subterráneo durante doce años, aparece en escena en el momento en que liberan a Diana, sin saber qué es la visión; diríase que ella va a asumir el truncado destino de la madre, ya que ésta sólo se comunica con ella ignorando al resto del mundo:

Alphonsine avait ce genre de beauté qui frappe tous les yeux et qui gagne tous les cœurs; plus on regardait son visage, plus on y trouvait de charme. De grands yeux d'un bleu foncé, ombragés de longues paupières noires; une peau d'une finesse incomparable, d'une blancheur extrême, sans aucun mélange de coloris; une tête toujours doucement inclinée, et parée d'une superbe chevelure blonde; une démarche nonchalante, un air craintif et timide, donnaient à toute sa personne la grâce la plus touchante, et un intérêt inexprimable. Elle parlait peu; elle avait toujours les yeux fixés sur sa mère, à laquelle elle obéissait au moindre mot (I: 192).

La educación recibida convierte a Alphonsine en una niña única:

La voilà, cette enfant, unique sur la terre par son innocence et par sa sensibilité! cet être angélique, dont les sens sont aussi purs que le cœur, dont toutes les sensations sont intimement unies à des sentiments vertueux! Élevée entre Dieu et celle qui lui donna la vie, son oreille n'a jamais entendu que les louanges de son Créateur et les douces instructions d'une mère, sa bouche n'a 
jamais proféré un mensonge ou une médisance; elle ne connaît l'orgueil que par l'histoire des anges rebelles; elle n'a pas l'idée de la vanité, de l'envie, de la jalousie, de la moquerie, de la colère... (II: 162).

En palabras de Alvar, «Grand Dieu! qu'elle est belle et touchante! et tout l'attrait de la pureté, toutes les grâces de l'innocence, sont réunis dans sa personne!» (II: 304), algo que más tarde augura lo que ocurrirá entre ellos:

De premier mouvement, comme par réflexion, elle me sacrifiera, sans hésiter, à son devoir, et elle croira ne faire qu'une action simple et naturelle. Sa pureté, sa tendresse filiale, la reconnaissance et la vertu, préservent à jamais son cœur des emportemens d'une passion violente; elle ne connaîtra de l'amour que ce qu'il peut inspirer de délicat et de généreux; il ne l'égarera point, parce qu'il ne la dominera jamais; il ne lui fera éprouver que de l'attendrissement et de la mélancolie; mais il ne détruira ni son repos ni son bonheur, tant qu'il sera combattu par son devoir (II: 305).

Alvar se maravilla continuamente de su pureza e inocencia:

Cette innocence parfaite, unique à son âge sur la terre, et qui la pare à mes yeux plus encore que sa beauté... Elle ne peut inspirer que des pensées attendrissantes et pures... Que j'aimais à voir le soleil éclairer cette figure ravissante, qui fut si longtemps cachée dans les ténèbres! Avec quel plaisir je contemplais ces beaux yeux, qui n'ont jamais entrevu le vice, ou même des spectacles profanes! (II: 306).

Alphonsine no ha conocido nada de la vida, dada su aparente ceguera al vivir en un sitio oscuro; diríase que la autora quiere demostrar que es precisa una educación sin contaminación alguna del exterior para las jóvenes, ya que Inès, a los dieciséis años, al desarrollarse su educación en la corte, ya ha perdido la ingenuidad. Aparece en la novela a comienzos del segundo tomo y es el modelo elegido por Genlis para demostrar una educación desprovista de artificios. Sus primeras palabras, de glorificación al Señor, a los diez meses son Mon Dieu!..., repetidas ante el asombro de lo que se presenta ante su vista (II: 32-33).

Poco a poco van desarrollándose en la niña los sentidos y es sensible al ruido, se ríe por primera vez, responde a las caricias aunque no llega a andar hasta tarde; no muestra vivacidad ni alegría, pero sí una extrema sensibilidad al acariciar a su madre, siendo muy perceptiva a los sonidos de la voz materna. El apego continuo y constante a su madre le proporcionaba una inteligencia en los sentimientos, superior a la que puede dar la vivacidad del espíritu. Recogiendo la transmisión de la madre, se acostumbra a una sumisión y perfecta obediencia desde la primera infancia. Finalmente, la niña es legitimada por su padre como Alphonsine d'Almédor. 
g) Alvar, el hijo de la condesa de Olmas y futuro marido de Alphonsine, es descrito de este modo:

La comtesse avait un fils unique de son premier mariage. Cet enfant, qui se nommait $\mathrm{D}$. Alvar, était alors âgé de six ans. La nature avait beaucoup fait pour lui; il était beau, spirituel et sensible; mais déjà gâté par le faste et par la flatterie. Il savait que le bien du feu duc son père lui assurait une fortune considérable, et qu'en outre on lui destinait pour épouse la plus riche héritière de l'Espagne; cette jeune personne, un peu plus âgée que lui, était orpheline, et se nommait dona Inès. La comtesse, sa parente et sa tutrice, s'était chargée de son éducation, et partageait son affection maternelle entre elle et don Alvar (I: 32).

Un retrato más completo lo encontramos en el capítulo xxxvi:

Don Alvar était beau, aimable, spirituel, vif, confiant; il avait des manières nobles et douces; il parlait avec facilité le français, l'anglais et l'allemand; la science de son mentor faisait supposer qu'il en avait beaucoup lui-même; on lui savait gré de n'en point montrer, et toutes les femmes assuraient que la frivolité et la légèreté n'étaient en lui que de la condescendance. Il eut donc de grands succès, et, pour cacher ses intrigues à M. Antonio, il n'eut besoin d'aucun effort d'imagination, ni même d'aucune adresse (II: 278).

Alvar, que ha desencadenado el primer sentimiento de pudor en Alphonsine, piensa que ella lo amará porque «ses yeux me l'ont promis»; es decir, por una curiosa similitud «voz/visión», parecería que la vista (que empieza a expresarse) le hablara a Alvar del amor de Alphonsine por él. La mirada de Alphonsine, desprovista de toda simulación o artificio, obra a modo de prolepsis en Alvar: «Elle m'aimera, ses yeux me l'ont promis; ce regard si chaste, si pur et si tendre, m’a dévoilé tout notre avenir...» (II: 189).

h) Inès la prometida de Alvar, que luego se casará con Dazeli; ya hemos visto como su educación en la corte la ha llevado a perder la ingenuidad. La escritora, describe su carácter en dos pasajes distintos:

Inès, âgée de seize ans, et ayant beaucoup été dans le grand monde, n’avait pas cette ingénuité, charme si doux de la première jeunesse; mais, remplie d'esprit, de finesse et de grâces, joignant aux agréments extérieurs un caractère ouvert, une âme sensible, une humeur toujours égale et gaie, elle faisait les délices de la comtesse, dont elle payait les soins par l'attachement le plus sincère et le plus tendre (I: 140-141).

Cette dernière était proche parente de la duchesse d'Alzuna, elle s'intéressait beaucoup à Dazeli; l'idée de ce mariage la charma. Elle fut, d'ailleurs, très-flattée de la confiance que lui montrait Dazeli, car elle aimait à être consultée: c'est le faible de toutes les personnes d'un esprit médiocre; c'est 
pourquoi, en général, elles ont du commérage et sont officieuses; une confidence est non-seulement pour elles une marque d'amitié, mais un honneur; elles sont glorieuses de pouvoir se mêler d'une affaire, de pouvoir contribuer à un succès (II: 251-252).

i) El mentor de Alvar, M. Antonio, trasunto de los filósofos y elegido por su madre entre miles, en viaje con su discípulo por Francia, Inglaterra, Suiza y Alemania, trata de formarlo en el estudio de las ciencias naturales, botánica, mineralogía y química, así como en el conocimiento de los habitantes de las naciones que visitan. La opinión sobre este modo de educar de la autora es clara y rotunda:

Tel était le mentor que la comtesse de Moncalde s'applaudissait d'avoir choisi entre mille; c'était, en effet, un savant distingué, et d'une conduite irréprochable; mais on eût beaucoup mieux fait de lui préférer un ignorant, qui aurait joint aux mêmes mœurs, de la vigilance, de l'esprit naturel, et un peu d'usage du monde (II: 276).

j) Don Juan de Oropésa:

Don Juan d'Oropésa, grand seigneur, jeune, aimable et passionné. (...) était l'homme de la cour le plus considéré, parce qu'il avait à la fois de la grandeur dans les sentiments, et de l'originalité dans l'esprit, ce qui réussit toujours avec de la fortune et un rang élevé. (...). Don Juan, âgé de trente-deux ans, avait une belle figure, un maintien froid et sévère; il était naturellement frondeur, et vivement frappé des vices et des travers de la société; il mettait son amour-propre à ne ressembler à personne. Il y parvenait sans effort, en suivant ses premiers mouvements, qui étaient toujours d'une générosité chevaleresque (II. 281-282).

\section{Dinámica narrativa}

La dinámica narrativa del texto se sustenta, por un lado, por el relato de la propia narradora-autora (Genlis); por otro, por otras narraciones y confesiones:

a) la narración de Dazeli al rey contándole su historia;

b) la narración del cura del pueblo cercano al castillo de Granada a la duquesa Olmas y segunda esposa del conde de Moncalde;

c) la confesión del propio conde de Moncalde a la justicia, por la que es desterrado a su castillo de Granada;

d) la confesión de Léonore a la duquesa Olmas, una vez muerto su marido el conde;

e) la narración de Doña Diana de Mendoce al ser liberada, llevada a cabo por medio del manuscrito que ha ido escribiendo en su cautividad. 
Basándose en estas narraciones, la autora va descubriendo nuevos secretos que el lector no sospechaba y que siempre superan su imaginación. Es de notar que la novela presenta una gran simetría entre la narración de la autora y las narraciones de Dazeli y Diana, que por su longitud ocupan casi la mitad del primer tomo, equivalente en extensión estos dos capítulos al resto de los anteriores.

Conforme va avanzando la novela, cada revelación de algún narrador o alguna confesión va poniendo de relieve la crueldad del conde de Moncalde, quien tiene todo planificado desde el mismo momento de su boda poniendo en antecedentes a su esposa de sus aviesas intenciones y se revela como un hombre duro, autoritario y despótico, sin principios, a la vez que vicioso, al que Diana piensa volver a la virtud a fuerza de dulzura, paciencia y ternura.

La simetría es tal que en ocasiones asimila la causa con su finalidad: Diana, que no ha visto consumado su matrimonio con Don Pèdre, el conde de Moncalde, verá consumada su relación con Don Pèdre de Almedor ${ }^{11}$ la misma fecha de la boda con el primero, el 1 de mayo.

Contribuyen a marcar la dinámica narrativa varias prolepsis o anticipaciones en el relato, utilizadas por el narrador para adelantar acontecimientos a los lectores. Así, la duquesa de Olmas ve la figura de su hermano reflejada en el agua, durante su estancia desgraciada en Granada.

Al huir de su hogar (según cuenta Dazeli), Diana tiene siniestros presentimientos:

Ah!... Dazeli, me dit-elle, je ne vais pas rejoindre le comte; je fais une démarche bien téméraire; je n'ai plus le droit de compter sur la protection du ciel; je suis accablée d'inquiétudes et de pressentiments sinistres! (I: 62).

Y más adelante: «Le ciel venait de se couvrir de nuages; la nuit était excessivement sombre» (I: 63). En la huida, Diana se da cuenta de que va hacia un camino de infelicidad:

C'en est fait, m'écriai-je, rien ne peut désormais me sauver; on m'entraîne sans retour loin de ma famille et de ma patrie... Ah! Si je n'avais à craindre que le malheur! Mais je cours vers la honte, et chaque pas semble accroître ma douleur et mon repentir... (I: 272).

La casi plena seguridad de su estancia en la cueva durante años es intuida tras tomar la decisión de no marcharse con Don Sanche; esa intuición le proporcionará a la vez un gran alivio y consuelo, ya que la religión le aportará serenidad ante la desesperación:

\footnotetext{
11 Es de notar la coincidencia silábica y casi ortográfica de los apellidos de los dos nobles.
} 
Un pressentiment secret m'avertissait que j'y passerais plusieurs années, que j’y élèverais paisiblement mon enfant, et qu'ensuite nous en sortirions avant que mon enfant eût atteint les premières années de la jeunesse. Cette idée est restée tellement gravée dans mon imagination et dans mon cœur, que sans chercher à deviner comment elle pourrait se réaliser, elle a toujours fait ma plus grande consolation (I: 351-352).

\section{LA COORDENADA ESPACIAL Y LAS DESCRIPGIONES DEL ESPACIO ${ }^{12}$}

Para numerosos teóricos de la novela el nivel espacial es solidario de los restantes niveles o materiales de la Historia, incluyéndolo, por esa razón, en el ámbito de ésta, según el esquema:

\section{Acontecimientos (funciones)}

Historia-Personajes (acciones)

Espacio $^{13}$

El espacio de la novela de Genlis se desarrolla casi siempre en España, salvo los viajes que llevan a cabo al extranjero Alvar con su mentor, M. Antonio, y el final de Diana y Alphonsine a Inglaterra, donde encuentran a su futuro marido y padre, Don Pèdre de Almédor.

España suele ser evocada de un modo positivo (aunque en dos de los lugares, Granada y Cádiz estén situados los castillos de los encierros de Dazeli y Diana). El recuerdo de Andalucía, y más concretamente el de Granada, parece estar siempre presente en la mente de Mme de Genlis; algo lógico si pensamos que la autora, por su condición de escritora de novelas históricas, conocía las novelas moriscas que tuvieron gran repercusión en la Francia del XVII ${ }^{14}$. Madrid, el lugar en que se inicia la acción, es evocada positivamente, sobre todo al asociar su recuerdo al de Don Pèdre de Almédor, así como

12 Una distinción básica del espacio nos lleva a diferenciar entre: Espacio geográfico, territorial (accidente)/Espacio literario (esencia). Sabido es que el espacio en la novela no tiene siempre vida propia, sino que a veces es «creado» por el personaje; esa creación revela un carácter de figuración simbólica. En estos casos de identificación absoluta y plena entre el espacio y los personajes, la atmósfera maligna del lugar llega casi a convertirse en otro personaje más y todos los elementos contenidos en esa atmósfera adquieren caracteres negativos: el aire está viciado o corrompido, la luz difusa o equívoca, el agua estancada o impura, los colores negros o sombríos, etc.; en resumen, todo se corresponde con la intención de la ficción que conduce a un equilibrio textual coherente.

13 Esta opción es fácilmente justificable ya que el espacio es el marco representativo de una referencialidad geográfica donde se instalan los personajes con sus actuaciones y conflictos, aunque también es verdad que el espacio de la novela es autónomo y suficiente.

${ }_{14}$ El relato «hispano-mauresque» es un tipo de narración inspirado en la novela morisca española del Xvi, que se difundiría en Francia durante todo el siglo Xvir llegando su influencia 
Granada, La Alhambra y los abencerrajes ${ }^{15}$. Generalmente España, cuando no es lugar de encierro, es espacio de felicidad: Lady Sarah, la inglesa de quien se enamora Don Pèdre de Almédor, se establece definitivamente en Madrid y Granada.

De un modo general se puede afirmar que el castillo, sobre todo cuando es lugar de encierro, es el espacio de la desdicha por oposición al campo abierto, espacio de la liberación y la felicidad; conforme los espacios abiertos se van haciendo más elevados la dicha va aumentando, en una asimilación a la subida a los cielos: el camino que conduce a la ermita donde bautizan a Alphonsine ${ }^{16}$.

Las estaciones están relacionadas con la felicidad/desgracia de los personajes sensibles: la primavera, la «belle saison», al ser la época en que se casa Diana con el duque de Moncalde, a pesar de su aspecto de felicidad, es una estación triste para ella, ya que le ayuda a recordar su desgracia, aunque a veces el corazón confunda los sentimientos.

El apoyo de la religión puede hacer que un espacio sombrío y funesto, como el castillo donde está encerrada Diana, pueda convertirse en un lugar de felicidad:

Il est vrai, me disais-je, qu'ici je suis privée de la lumière du soleil et de la contemplation de la nature; mais Dieu n'est-il pas ici comme dans les déserts? n'ai-je pas l'espoir d'y doubler mon existence? O! si la bonté suprême produit un tel miracle, cette caverne deviendra le temple de la reconnaissance et du bonheur!... (r: 327-328).

Una vez terminado el bautismo de Alphonsine, y dirigiéndose los fieles al castillo, éste deja de ser estancia de terror y espanto, ya que su habitante principal, Alphonsine, está iluminado por la fe católica:

On voulut retourner au château avant que le soleil eût pris toute son ardeur; Diana, la comtesse, Alphonsine, Inès et Dazeli, montèrent ensemble dans une calèche ouverte des côtés, et l'on prit le chemin du château. Alphonsine, durant cette course, ne montra pas la moindre frayeur (II: 228).

No obstante, para que el castillo deje de ser tenebroso y triste, hay que «abrirlo al exterior», de ahí la simbología del gesto de Alphonsine abriendo

\footnotetext{
hasta finales del xvin con el fabulista e hispanista Florian, y finalizaría con Les Aventures du dernier Abencérage de Chateaubriand, en 1826.

15 El nombre de Abencerrajes proviene del apellido de una familia de la nobleza de la época, que vivía en el interior de la Alhambra.

${ }_{16}$ Para ser feliz, la condesa sale del castillo y se pasea por el campo. La boda de Diana se celebra en el campo a dos leguas de Madrid, según relata la propia Diana en su manuscrito.
} 
las ventanas: «Arrivée au château, Alphonsine courut dans sa chambre pour en ouvrir les fenêtres, qui donnaient sur le jardin: cette vue nouvelle lui parut admirable» (II: 229). En ocasiones el espacio va asociado a las descripciones del paisaje:

Les deux jours d'ensuite, elle lit de longues promenades dans le parc, qui était extrêmement négligé, mais très spacieux. La veille de son départ, elle se leva avec le jour, et descendit aussitôt dans le jardin; elle fut s'asseoir sur un banc placé sur le bord d'une espèce de torrent, formé par une fontaine naturelle, qui se précipitait du haut d'un énorme rocher, caché en grande partie par des cyprès et des grenadiers nouvellement plantés (I: 40).

El paisaje cuenta en el libro con acertadas descripciones, ya que es la obra del Creador, sirviendo a veces de consuelo a los personajes virtuosos. Otras veces, el descubrimiento del paisaje hace que en Alphonsine estallen todas las emociones contenidas hasta ese momento:

Il fallut gravir une montagne assez escarpée. Parvenue au sommet on s'arrêta. O mon enfant, dit Diana, nous sommes arrivées! A ces mots Alphonsine fit éclater la joie la plus touchante; elle pleurait, elle tremblait, elle embrassait sa mère, son cœur palpitait avec une telle violence, que Diana voulut attendre qu'elle fût un peu calmée avant de la laisser descendre de voiture (II: 218).

El espectáculo de la naturaleza en toda su belleza, en el momento en que Alphonsine puede contemplarla, es una de las páginas más brillantes de la novela, acercando a los seres al Sumo Hacedor:

Mon Alphonsine, s'écria Diana, fais-moi jouir enfin des beautés de l'univers éclipsées pour moi depuis tant d'années; rends à ton heureuse mère la lumière céleste et la nature; ouvre les yeux, et regarde devant toi... A ces mots, Diana, se débarrassant de son voile, place Alphonsine en face de l'allée d'orangers; Alphonsine fait un cri de surprise et d'admiration; sa mère aussitôt, la soutenant dans ses bras, la fait retourner de l'autre côté, et lui montre à-la-fois la vallée le fleuve, la forêt, et le soleil levant... (II: 220).

Este espectáculo de la naturaleza, recién descubierta por Alphonsine, que conduce a la iglesia, es el complemento necesario para la religión:

Instruite des mystères de la religion, elle se trouvait pour la première fois de sa vie dans le sanctuaire du vrai Dieu, avec toute son innocence et toute sa raison: le spectacle de la nature, qui venait tout-à-coup de se dévoiler à ses yeux, était tout-à-la-fois pour elle une découverte merveilleuse, et la preuve la plus frappante des vérités religieuses. Sa foi s'était accrue encore par son admiration; au milieu de tant de prodiges, qu'elle n'ignorait pas que la science humaine ne saurait expliquer, son esprit recevait sans obstacles toutes les 
clartés divines de la révélation, et les mystères de la religion lui paraissaient beaucoup moins étonnants que ceux de la nature (II: 223-224).

En un caso específico, el encierro de Diana y Alphonsine es la representación de la alegoría de la caverna platónica:

Quand elle m'a demandé si le monde où nous sommes est l'univers tout entier, je lui ai répondu qu'il y a encore d'autres parties du monde habitées par d'autres créatures, mais qu'il ne nous est pas possible de communiquer avec ces êtres semblables à nous, parce qu'ils sont trop éloignés de notre monde (II: 136).

\section{Conclusión}

Mme de Genlis, que en un principio había decidido como título para su novela l'Éducation sensitive, ou la Tendresse maternelle, ya que este título expresaba perfectamente la idea de fondo del relato, creía, al contrario que Rousseau, que hay espiritualidad incluso en nuestros sentidos y que el alma los ennoblece al ampliar su uso. Todas las facultades que Dios ha dado al hombre sirven para que logre la felicidad, que se llega a alcanzar por medio de hechos y no de razonamientos. La intención de la autora es mostrar que, hasta en la situación más deplorable, las almas virtuosas y sensibles están dotadas de recursos infinitos que las pueden ayudar a salir de su infierno, de ahí que revista a su heroína de un gran candor, pureza e inocencia, lo que la lleva a mantenerla alejada de un verdadero amor carnal, que agitaría su corazón con toda la violencia imaginable. Los retratos de los personajes que pueblan sus novelas demuestran generalmente un profundo conocimiento de las almas, tanto femeninas como masculinas, constituyendo en ocasiones hondas y atinadas reflexiones sobre la naturaleza humana.

Para poner de relieve sus ideas Mme de Genlis se sirve de un léxico formado por vocablos pertenecientes a un registro culto, siendo el denominador común de estos textos los sustantivos abstractos y denotados, que empleados en estructuras sintácticas recurrentes (oraciones condicionales, estructuras impersonales que indican obligación, imperativos) contribuyen a la insistencia del tema tratado, dando coherencia al texto.

Curioso caso el de Mme de Genlis, conocida en nuestros días por ser la autora de Adèle et Théodore, ou Lettres sur l'éducation (1782) y por Veillées du château, ou cours de morale à l'usage des enfants (1784), antología de apólogos y cuentos morales que serían traducidos al inglés, al alemán y al español. Se puede afirmar que la posteridad únicamente ha considerado estos dos libros pedagógicos de una obra de más cien volúmenes. A pesar de esta constatación, una lectura atenta de algunas de sus obras muestra que no sólo los libros sobre educación, sino también sus relatos históricos atesoran un mérito 
indiscutible ajustándose tanto como los otros a los cánones normativos de ese tipo de obras. En este sentido, Alphonsine es una buena conjunción de los valores intrínsecos de ambos.

La crítica de su época la denigraría y detestaría antes que reconocerle cualidades de escritora, no aceptando de buen grado la enorme variedad de sus libros, pues era práctica normal en ella que publicara tanto una obra de teatro para niños, como una obra ascética, novelas históricas, tratados de pedagogía, etc. A este respecto, es reveladora la opinión de Rémy Landy cuando habla del rechazo hacia nuestra autora del escritor y periodista francés, y sobre todo polemista y adversario de la Revolución, Antoine de Rivarol:

Il faut donc refuser, à propos de Mme de Genlis, des jugements aussi péremptoires que celui de son ennemi Rivarol: «Le ciel refusa la magie du talent à ses productions, comme le charme de l'innocence à sa jeunesse» (Landy, 1998: 966).

Juicio inmerecido a todas luces sobre una escritora que sufriría un injusto olvido después de su muerte ${ }^{17}$, a pesar de haber combinado, no sin habilidad, las técnicas de Diderot y de Mme de La Fayette o de La Bruyère, para lograr trazar el carácter del equivalente de un René femenino con Inès de Castro, construyendo narraciones históricas que pueden considerarse en algunos aspectos como un precedente de las de Alejandro Dumas.

\section{BiBLIOGRAFÍA}

Barthélemy, J.G. (2006): Stéphanie Félicité, comtesse de Genlis. Paris, La Société des écrivains.

Bessire, F. y Reid, M. (dirs.) (2008): Madame de Genlis. Littérature et éducation. MontSaint-Aignan, Publications des Universités de Rouen et du Havre.

Broglie, G. (2001 [1985]): Madame de Genlis. Paris, Perrin.

Díaz Canseco, V. (1844): Diccionario biográfico universal de mujeres célebres. Madrid, Imprenta de D. José Félix Palacios.

F[ERNÁNDEZ] Montesinos, J. (1982): Introducción a una historia de la novela en España en el siglo XIX. Seguida del esbozo de una bibliografía española de traducciones de novelas (1800-1850). 4. ${ }^{\text {a }}$ ed. Madrid, Editorial Castalia.

Genlis, Mme de (1806a): Alphonsine ou la Tendresse Maternelle, 2 vols. Paris, Nicolle et Compagnie.

- (1806b): Alphonsine: or, Maternal Affection. A Novel, 4 vols. London, Printed for J.F. Hughes.

17 Gustave Lanson ni siquiera la mencionaría en su Histoire de la littérature française (1895). 
Hervey, S. y Higgins, I. (1992): Thinking translation. A course in translation method: French to English. London-New York, Routledge.

Kavanagh, J. (1862): French Women on Letters. Biographical Sketches, 2 vols. London, Hurst and Blackett Publishers.

Laborde, A.M. (1966): L'Euvre de Mme de Genlis. Paris, Nizet.

Landy, R. (1998): «Mme de Genlis». En Beaumarchais, J.P. et al. (eds.): Dictionnaire des littératures de langue française, vol. II: E-L. Paris, Bordas, págs. 965-966. 